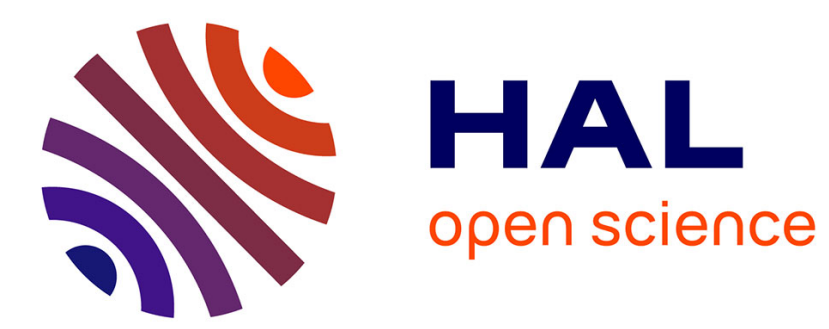

\title{
On Homogeneous Evolution Equation in a Banach Space
} Andrey Polyakov, Denis Efimov, E Fridman, Wilfrid Perruquetti

\section{To cite this version:}

Andrey Polyakov, Denis Efimov, E Fridman, Wilfrid Perruquetti. On Homogeneous Evolution Equation in a Banach Space. European Control Conference 2015, Jul 2015, Linz, Austria. hal-01160068

\section{HAL Id: hal-01160068 \\ https://hal.inria.fr/hal-01160068}

Submitted on 4 Jun 2015

HAL is a multi-disciplinary open access archive for the deposit and dissemination of scientific research documents, whether they are published or not. The documents may come from teaching and research institutions in France or abroad, or from public or private research centers.
L'archive ouverte pluridisciplinaire HAL, est destinée au dépôt et à la diffusion de documents scientifiques de niveau recherche, publiés ou non, émanant des établissements d'enseignement et de recherche français ou étrangers, des laboratoires publics ou privés. 


\title{
On Homogeneous Evolution Equation in a Banach Space
}

\author{
A. Polyakov, D. Efimov, E. Fridman, W. Perruquetti
}

\begin{abstract}
A geometric homogeneity of evolution equation in a Banach space is introduced. Scalability property of solutions of homogeneous evolution equations is proven. Some qualitative characteristics of stability of trivial solution are also provided. In particular, finite-time stability of homogeneous evolution equations is studied. Theoretical results are supported by examples from mathematical physics.
\end{abstract}

\section{INTRODUCTION}

The homogeneity is a sort of symmetry, when an object remains consistent (in some sense) with respect to a scaling operation (dilation). In the context of ordinary differential equations and inclusions one encounters three types of homogeneity:

- the standard homogeneity (L. Euler in the 17th century, V. Zubov [43], W. Hahn [17]) operates with uniform dilations such as $x \rightarrow \lambda x$, where $\lambda>0$ is a real number and $x$ is an element of a real linear space;

- the weighted homogeneity (V. Zubov [44], H. Hermes [19], L. Rosier [33]) uses non-uniform (anisotropic) scalings:

$$
\left(x_{1}, x_{2}, \ldots, x_{n}\right) \rightarrow\left(\lambda^{r_{1}} x_{1}, \lambda^{r_{2}} x_{2}, \ldots, \lambda^{r_{n}} x_{n}\right),
$$

where $\lambda$ and $r_{i}$ are positive reals, $x_{i}$ is an element of a real linear space, $i=1,2, \ldots, n$;

- the geometric homogeneity (V. Khomenyuk [23], M. Kawski [22], A. Bacciotti \& L. Rosier [5]) considers some generalized dilations of one vector field with respect to another one.

Homogeneity is a useful tool for advanced analysis of nonlinear dynamic systems. For instance, it allows local properties of a system (e.g. asymptotic stability) to be extended globally. Qualitative stability analysis of homogeneous systems can be enhanced by means of investigation of homogeneity degree of an asymptotically stable system, for example, the negative degree corresponds to finite-time stability [43], [11], [27], [25], [5], [3]. The finite-time stability is also known as super-stability [41] , [39], [2]. The control theory applies homogeneous feedbacks for fast robust stabilization (see, [18], [11], [25], [3], [32] and homogeneous dynamic observers for non-asymptotic state estimation [29], [30]. Homogeneity provides simple algorithms for robustness analysis of nonlinear control systems (in the context of Input-to-State Stability see, for example, [35], [20], [9]). Local homogeneity and homogeneous approximations [44],

A. Polyakov, D. Efimov and W. Perruquetti are with Inria Lille-Nord Europe, 40, avenue Halley, 59650 Villeneuve d'Ascq, France and with CRIStAL UMR CNRS 9189, 59650 Villeneuve d'Ascq, France. E. Fridman is with School of Electrical Engineering at Tel-Aviv State University, Ramat Aviv, Tel Aviv 69978, Israel
[19], [3], [16] are considered as a way for simplification of qualitative analysis of the essentially nonlinear dynamic systems. It is worth to stress that investigations of quantitative characteristics (for example, estimation of the settling time for finite-time stable system) requires conventional tools (e.g. Lyapunov function method). However, it is well known [44], [33] that any stable homogeneous systems always has a homogeneous Lyapunov function. This property simplifies the construction of concrete explicit [38], [5], [26] and implicit Lyapunov functions [31] for homogeneous systems.

The analysis of evolution equations with homogeneous (with respect to uniform scaling) operator was given in [7]. Some important regularizing effects of homogeneity have been discovered in the mentioned paper. Weighted homogeneous evolution equations have been studied in [37], [40]. The most of results for such systems are devoted to the integrability analysis. To the best of our knowledge, stability properties (in particular, finite-time stability) of evolution equation have not been studied using homogeneity framework. In the same time, these issues are very important for control and estimation problems for distributed parameters systems [28].

In this paper, we introduce the notion of $\mathbf{d}$-homogeneous operator in a Banach space, where $\mathbf{d}$ denotes a semigroup of homogeneous dilations. This type of homogeneity can be considered as a certain analog of geometric homogeneity known for finite-dimensional vector fields. We show that many important stability properties of the homogeneous finite-dimensional systems can be observed for dhomogeneous evolution equations in Banach spaces. The presented results can be utilized in future for an expansion of homogeneous feedback control design methods to evolution equations.

The paper is organized as follows. The next section presents notations to be used in the paper. Model description and basic assumptions are given in the section 3. The section 4 introduces the notion of homogeneous evolution equation in Banach space. It presents the generalized dilation semigroup, the homogeneous set and the homogeneous operator, and studies their properties. Finally, some concluding remarks finish the paper.

\section{NOTATIONS}

- $\mathbb{R}$ is the field of real numbers and $\mathbb{R}_{+}=(0,+\infty)$;

- $\mathbb{B}$ is a real Banach space with a norm $\|\cdot\|$;

- $S=\{u \in \mathbb{B}:\|u\|=1\}$ is the unit sphere in $\mathbb{B}$;

- $B=\{u \in \mathbb{B}:\|u\|<1\}$ is the unit ball in $\mathbb{B}$;

- $\mathbb{C}(\mathbb{X}, \mathbb{Y})$ is the space of continuous functions $\mathbb{X} \rightarrow \mathbb{Y}$, where $\mathbb{X}, \mathbb{Y}$ are some appropriate sets; 
- $\mathbb{C}^{r}(\mathbb{X}, \mathbb{Y})$ is the space of continuous functions $\mathbb{X} \rightarrow$ $\mathbb{Y}$, which have continuous derivatives at least up to the order $r$;

- $\mathbb{L}^{q}(\mathbb{X}, \mathbb{Y})$ is the space of functions $\mathbb{X} \rightarrow \mathbb{Y}$, for which the q-th power of the absolute value is Lebesgue integrable;

- $\bar{\Omega}$ denotes the closure of the set $\Omega \subset \mathbb{B}$;

- $\partial \Omega$ denotes the boundary of the set $\Omega \subset \mathbb{B}$.

\section{MODEL DESCRIPTION}

Let us consider the nonlinear evolution equation

$$
\dot{u}(t)=f(u(t)), \quad t \in \mathbb{R}_{+}, \quad u(\cdot) \in \Omega \subset \mathbb{B}
$$

with the initial condition

$$
u(0)=\varphi \in \Omega,
$$

where the dot denotes a time derivative, the operator $f: D \subset$ $\mathbb{B} \rightarrow \mathbb{B}$ has the domain $D$ and $\Omega \subset D$

Recall that evolution equation may describe finite or infinite dimensional dynamic system. The set $\Omega$ restricts admissible solutions. For example, if it is a positive cone (i.e. $\Omega=\mathbb{R}_{+}^{n}$ ), the evolution equation must describe positive system. In the case of a partial differential equation the set $\Omega$ can be utilized in order to possess boundary conditions.

We assume that the Cauchy problem (1), (2) has a solution for any $\varphi \in \Omega$, i.e. there exists a continuous function $u:[0, T) \rightarrow \Omega$ defined at least locally $(0<T \leq+\infty)$, which satisfies (in some sense) the equation (1) and the initial condition (2). We do not specify the type of solution. It can be classical $\left(\mathbb{C}^{1}\right)$, strong, weak or mild solution. Proofs of main results are given for mild solutions. The extension to other types of solutions is straightforward. The same results can be provided for evolution inclusions.

In general, we do not assume that the Cauchy problem is well-posed, i.e. the solutions may be non-unique and/or they may not depend continuously on the initial condition. Certainly, the results to be obtained also hold for well-posed models. Existence of solutions and well-posedness of the Cauchy problem like (1), (2) are discussed in literature for different types of operators $f$ and Banach spaces $\mathbb{B}$, see e.g. [1], [42], [6]. These problems go out of the scope of this paper. The main aim is to extend the concept of homogeneous systems to the general evolution equations and to transfer some useful properties and tools, which are derived for homogeneous ordinary differential equations, to a more general dynamical models.

\section{Homogeneous Evolution Equations And Their PROPERTIES}

The evolution equation (1) is uniquely identified by the operator $f$ and the set $\Omega$. Having the same operator $f$ : $D \subset \mathbb{B} \rightarrow \mathbb{B}$ for different sets $\Omega_{1} \subset D$ and $\Omega_{2} \subset D$, $\Omega_{1} \neq \Omega_{2}$ we may obtain different behavior of solutions of evolution equation. Therefore, in the context of the evolution equations the homogeneity properties must be studied for both the operator $f$ and the set $\Omega$.

\section{A. Homogeneous Sets in Banach Spaces}

Let $\mathcal{L}(\mathbb{B})$ be the space of linear bounded operators $\mathbb{B} \rightarrow \mathbb{B}$ equipped with the norm: $\|g\|_{\mathcal{L}}=\sup _{u \in S}\|g(u)\|$ for $g \in \mathcal{L}(\mathbb{B})$.

Definition 1: A map $\mathbf{d}: \mathbb{R} \rightarrow \mathcal{L}(\mathbb{B})$ is called dilation in the space $\mathbb{B}$ if it satisfies

- the semigroup property: $\mathbf{d}(0)=I \in \mathcal{L}(\mathbb{B})$ and $\mathbf{d}(t+$ $s)=\mathbf{d}(t) \mathbf{d}(s)$ for $t, s \in \mathbb{R}$;

- the strong continuity property: the map $\mathbf{d}(\cdot) u: \mathbb{R} \rightarrow$ $\mathbb{B}$ is continuous for any $u \in \mathbb{B}$;

- the limit property: $\lim _{s \rightarrow-\infty}\|\mathbf{d}(s) u\|=0$ and $\lim _{s \rightarrow+\infty}\|\mathbf{d}(s) u\|=\infty$ uniformly on $u \in S$.

The dilation $\mathbf{d}$ is the strongly continuous group, since the limit property implies uniqueness of the identity element (see, Proposition 1 given below). The group $\mathbf{d}$ has similar topological characterization as dilation mappings in Banach (or Frechet) spaces [21]. The limit property given above can be interpreted as a version of the Teresaka's condition (see, for example, [21]).

Proposition 1: If $\mathbf{d}$ is a dilation then

1) $\|\mathbf{d}(s)\|_{\mathcal{L}} \neq 0$ for $s \in \mathbb{R}$ and $\mathbf{d}(s) \neq I \in \mathcal{L}(\mathbb{B})$ for $s \neq 0$;

2) $\inf _{u \in S}\|\mathbf{d}(s) u\|<1$ for $s<0$ and $\sup _{u \in S}\|\mathbf{d}(s) u\|>1$ for $s>0$. Proof. 1) The positivity of the operator norm of the dilation immediately follows from the semigroup property. Indeed, if there exists $s_{0} \in \mathbb{R}$ such that $\left\|\mathbf{d}\left(s_{0}\right)\right\|_{\mathcal{L}}=0$ then $\mathbf{d}\left(s_{0}\right)=$ $0 \in \mathcal{L}(\mathbb{B})$ and $I=d(0)=d\left(s_{0}-s_{0}\right) \neq d\left(-s_{0}\right) d\left(s_{0}\right)=0$.

In order to prove that identity element is unique let us suppose the contrary, i.e. there exists $s^{\prime} \in \mathbb{R} \backslash\{0\}$ such that $\mathbf{d}\left(s^{\prime}\right)=I$. Then the semigroup property implies $\mathbf{d}\left(n s^{\prime}\right)=I$ and $\left\|\mathbf{d}\left(n s^{\prime}\right) u\right\|=1$ for any $n=1,2, \ldots$ and any $u \in S$. This contradicts the limit property.

2) Let us prove the second claim. Consider, initially, the case $s \in \mathbb{R}_{+}$. Show that sufficient to show that $\|\mathbf{d}(s)\|_{\mathcal{L}}>1$ for $s>0$.

Suppose the contrary, i.e. there exists $s_{0} \in \mathbb{R}_{+}$such that $\left\|\mathbf{d}\left(s_{0}\right)\right\|_{\mathcal{L}} \leq 1$. In this case $\lim _{n \rightarrow+\infty}\left\|\mathbf{d}\left(n s_{0}\right) u\right\|_{\mathcal{L}}=+\infty$ for any $u \in S$ due to the limit property. On the other hand,

$$
\begin{gathered}
\left\|\mathbf{d}\left(n s_{0}\right) u\right\|=\left\|\mathbf{d}\left(s_{0}+(n-1) s_{0}\right) u\right\|=\left\|\mathbf{d}\left(s_{0}\right) \mathbf{d}\left((n-1) s_{0}\right) u\right\| \leq \\
\left\|\mathbf{d}\left(s_{0}\right)\right\|_{\mathcal{L}} \cdot\left\|\mathbf{d}\left((n-1) s_{0}\right) u\right\| \leq \ldots \leq\|u\| \leq 1,
\end{gathered}
$$

i.e. the sequence $\left\|\mathbf{d}\left(n s_{0}\right) u\right\|$ is bounded for any $n$ and we obtain the contradiction.

Consider the case $s<0$ and suppose the contrary, i.e. there exists $s_{0} \in \mathbb{R}_{+}$such that $\left\|\mathbf{d}\left(-s_{0}\right) u\right\| \geq 1$ for any $u \in S$. If $u_{0} \in S$ then

$$
u_{n}=\frac{\mathbf{d}\left(-s_{0}\right) u_{n-1}}{\left\|\mathbf{d}\left(-s_{0}\right) u_{n-1}\right\|} \in S, \quad n \geq 1 .
$$

So, we have

$$
\begin{aligned}
1 \leq\left\|\mathbf{d}\left(-s_{0}\right) u_{n}\right\| & =\left\|\frac{\mathbf{d}\left(-2 s_{0}\right) u_{n-1}}{\left\|\mathbf{d}\left(-s_{0}\right) u_{n-1}\right\|}\right\| \leq\left\|\mathbf{d}\left(-2 s_{0}\right) u_{n-1}\right\| \leq \\
& \ldots \leq\left\|\mathbf{d}\left(-(n+1) s_{0}\right) u_{0}\right\| .
\end{aligned}
$$

Therefore, $\left\|\mathbf{d}\left(-(n+1) s_{0}\right) u_{0}\right\| \geq 1$ for any $n \geq 1$ and we derive the contradiction to the limit property. 
The next definition introduces a class of domains of evolution operators to be studied in this paper.

Definition 2: A nonempty set $\Omega$ is said to be $\mathbf{d}$ homogeneous iff $\mathbf{d}(s) \Omega=\Omega$ for any $s \in \mathbb{R}$, where $\mathbf{d}$ : $\mathbb{R} \rightarrow \mathcal{L}(\mathbb{B})$ is a dilation in $\mathbb{B}$.

A d-homogeneous set $\Omega$ becomes a homogeneous space [4], if the dilation $\mathbf{d}$ is a transitive group on $\Omega$. The operators $\mathbf{d}(s)$ for $s \in \mathbb{R}$ are called symmetries in this case.

Let us give some examples of $\mathbf{d}$-homogeneous sets and their dilations.

- Uniform dilation (L. Euler, 17th century):

$$
\Omega=\mathbb{C}([0, p], \mathbb{R}), \mathbf{d}(s)=e^{s} \text {. }
$$

- Weighted dilation (Zubov 1958, [44]): $\Omega=\mathbb{R}^{n}, \mathbf{d}(s)=\operatorname{diag}\left\{e^{r_{i} s}\right\}, r_{i}>0, i=1,2, \ldots, n$.

- Geometric dilation (Kawski 1995, [22] and Khomenuk 1961, [23]): $\Omega=\mathbb{R}^{n}, \mathbf{d}$ is the flow of an Euler vector field ${ }^{1}$

- Generalized dilation in a Banach space:

$\Omega=\left\{u \in \mathbb{C}([0, p], \mathbb{R}): u(0)=u^{2}(p)\right\}$ and $(\mathbf{d}(s) u)(x)=e^{s-0.5 s x / p} u(x)$, where $x \in[0, p]$. Indeed, $(\mathbf{d}(s) u)(0)=e^{s} u(0)$ and $(\mathbf{d}(s) u)(p)=e^{0.5 s} u(p)$ imply $(\mathbf{d}(s) u)(0)=[(\mathbf{d}(s) u)(p)]^{2}$ for any $u \in \Omega$ and any $s \in \mathbb{R}$.

Note that $\boldsymbol{d}$-homogeneity can be considered as an analog of geometric homogeneity known for finite-dimensional vector fields. Indeed, in particular case, a group d may be generated by some evolution equation in the Banach space $\mathbb{B}$. This evolution equation must have unique solution globally defined on $\mathbb{R}$ for any initial condition. In addition, its zero solution has to be globally uniformly asymptotically stable in a backward time and all other solutions have to be unbounded in a forward time.

Homogeneous sets have some useful properties to be utilized below for analysis of evolution equation (1). For instance, if a nonempty set $\Omega$ is $\mathbf{d}$-homogeneous then $0 \in \bar{\Omega}$. Indeed, since $\|d(s) x\| \rightarrow 0$ as $s \rightarrow-\infty$ and $d(s) x \in \Omega$ for any $s \in \mathbb{R}$ and $x \in \Omega$ then any neighborhood of zero contains vectors from $\Omega$.

The set

$$
S_{\mathbf{d}}(r)=\{u \in \Omega:\|\mathbf{d}(\ln (r)) u\|=1\}, \quad r \in \mathbb{R}_{+}
$$

is called the homogeneous sphere of the radius $r$. Obviously, $S_{\mathbf{d}}(1)=\Omega \cap S$.

Proposition 2: The set $S_{\mathbf{d}}(1)$ is non-empty for any dhomogeneous set $\Omega \neq\{0\}$ and

- $S_{\mathbf{d}}(r)=\mathbf{d}(\ln (1 / r)) S_{\mathbf{d}}(1)$ for $r \in \mathbb{R}_{+}$;

- $\Omega \backslash\{0\}=\bigcup_{r \in \mathbb{R}_{+}} S_{\mathbf{d}}(r)$.

Proof. Let $u$ be an arbitrary element of the nonempty set $\Omega \backslash\{0\}$. If $\|u\|=1$ then $u \in S_{\mathbf{d}}(1)$. Since the group d is strongly continuous then the limit property implies that for $\|u\|>1$ there exists $s<0:\|\mathbf{d}(s) u\|=1$, and for $\|u\|<1$ there exists $s>0:\|\mathbf{d}(s) u\|=1$. Obviously, if $u \in \Omega$ and $u_{0} \in S_{\mathbf{d}}(1)$ is such that $\mathbf{d}(s) u=u_{0}$ then the semigroup property guarantees $\mathbf{d}(-s) \mathbf{d}(s) u=\mathbf{d}(-s) u_{0}$

\footnotetext{
${ }^{1}$ A $\mathbb{C}^{1}$ vector field $\nu: \mathbb{R}^{n} \rightarrow \mathbb{R}^{n}$ is called Euler if it is complete and $-\nu$ is globally asymptotically stable.
}

or equivalently $u=\mathbf{d}(-s) u_{0}$. Therefore, the inclusion $u \in$ $S_{\mathbf{d}}(r)$ implies that $z:=\mathbf{d}(\ln (r)) u \in S_{\mathbf{d}}(1)$ or equivalently $u=\mathbf{d}(-\ln (r)) z \in \mathbf{d}(\ln (1 / r)) S_{\mathbf{d}}(1)$.

\section{B. Homogeneous Operator in a Banach Space}

The definition given below presents the class of operators to be studied in this paper. It utilizes the conventional identity (4) in order to introduce the homogeneity relation (see, for example, [8]).

Definition 3: An operator $f: D \subset \mathbb{B} \rightarrow \mathbb{B}$ is said to be d-homogeneous of degree $\nu$ on the set $\Omega \subset D$ if $\Omega$ is d-homogeneous and

$$
f(\mathbf{d}(s) u)=e^{\nu s} \mathbf{d}(s) f(u) \quad s \in \mathbb{R}, \quad u \in \Omega,
$$

where $\mathbf{d}$ is a dilation in $\mathbb{B}$ and $\nu \in \mathbb{R}$.

The evolution equation (1) is said to be d-homogeneous on $\Omega$ if the corresponding operator $f: D \subset \mathbb{B} \rightarrow \mathbb{B}$ is d-homogeneous on $\Omega$.

Homogeneity can be discovered in many physical models. Examples of homogeneous ordinary differential equations can be found in the literature, see e.g. [44], [27], [29], [25]. Let us consider two examples of homogeneous partial differential equations, which appear in mathematical physics.

- Korteweg-de Vries equation ( $\mathrm{KdV}$ equation) is the classical example of the homogeneous partial differential equation ([24], [40]):

$$
\frac{\partial v}{\partial t}=-\frac{\partial^{3} v}{\partial x^{3}}-v \frac{\partial v}{\partial x},
$$

where $v$ is a scalar function of time $t \in \mathbb{R}_{+}$and space $x \in \mathbb{R}$ variables. $\mathrm{KdV}$ equation describes waves on shallow water surfaces. The operator $f: \mathbb{C}^{3}(\mathbb{R}, \mathbb{R}) \rightarrow$ $\mathbb{C}(\mathbb{R}, \mathbb{R})$ defined by $f(u)=-u^{\prime \prime \prime}-u u^{\prime}$ for $u \in$ $\mathbb{C}^{3}(\mathbb{R}, \mathbb{R})$ is d-homogeneous of degree $\nu=3$ with the dilation group defined by $(\mathbf{d}(s) u)(x)=e^{2 s} u\left(e^{s} x\right)$, where $x \in \mathbb{R}, u \in \mathbb{C}^{3}(\mathbb{R}, \mathbb{R})$ and $s \in \mathbb{R}$ is the dilation argument. Indeed, since $\left(d(s) u^{\prime}\right)(x)=\left.e^{2 s} u^{\prime}(y)\right|_{y=e^{s} x}$ and $[d(s) u]^{\prime}(x)=\left[e^{2 s} u\left(e^{s} x\right)\right]^{\prime}=\left.e^{3 s} u^{\prime}(y)\right|_{y=e^{s} x}$ for $x \in \mathbb{R}$ we derive

$$
\begin{aligned}
& {[f(d(s) u)](x)=-\left[e^{2 s} u\left(e^{s} x\right)\right]^{\prime \prime \prime}-e^{2 s} u\left(e^{s} x\right)\left[e^{2 s} u\left(e^{s} x\right)\right]^{\prime}=} \\
& -e^{5 s} u^{\prime \prime \prime}(y)-\left.e^{5 s} u(y) u^{\prime}(y)\right|_{y=e^{s} x}=\left[e^{3 s} d(s) f(u)\right](x),
\end{aligned}
$$

for any $s \in \mathbb{R}$.

- The Saint-Venant equation is an example of a system of conservation laws studied in [14]. In the field of hydraulics, it represents the flow in open-channels by the following model

$$
\begin{gathered}
\frac{\partial H}{\partial t}=-\frac{\partial}{\partial x}(H V), \\
\frac{\partial V}{\partial t}=-\frac{\partial}{\partial x}\left(\frac{1}{2} V^{2}+g H\right),
\end{gathered}
$$

where $H$ and $V$ are scalar functions of time and space variables. The quantity $H(t, x)$ is the water level at the instant of time $t \in \mathbb{R}_{+}$in the point $x \in \mathbb{R}$, and $V(t, x)$ is the water velocity in the same position. The parameter $g$ denotes the gravitation constant. Let us 


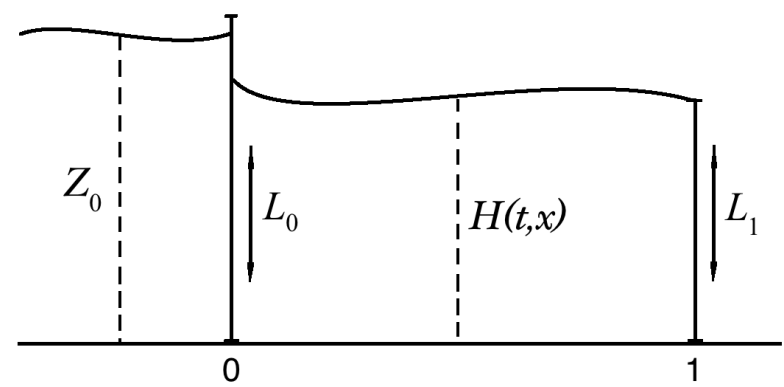

Fig. 1. Water channel with two spillways

consider the case when the water channel is supported by two overflow spillways (see, Figure 1), which adjusts an input and output flows in a pool (between spillways). In this case the space argument is restricted on the segment $[0,1]$, where $x=0$ and $x=1$ are positions spillways, and the equation (5) is supported with the boundary conditions [14]:

$$
\begin{gathered}
H(t, 0) V(t, 0)-\left(Z_{0}-L_{0}\right)^{3 / 2}=0, \\
H(t, 1) V(t, 1)-\left(H(t, 1)-L_{1}\right)^{3 / 2}=0,
\end{gathered}
$$

where $Z_{0}$ is the water level above the pool and $L_{0}, L_{1}$ are spillways.

Let us show that for $L_{0}=Z_{0}$ and $L_{1}=0$ the corresponding evolution equation is homogeneous. Let us consider the operator $f: D \rightarrow \mathbb{C}([0,1], \mathbb{R}) \times \mathbb{C}([0,1], \mathbb{R})$ defined on $D=\mathbb{C}^{1}\left([0,1], \mathbb{R}_{+}\right) \times \mathbb{C}^{1}([0,1], \mathbb{R})$ by $f(u)=\left(\begin{array}{c}-\frac{\partial}{\partial x}\left(u_{1} u_{2}\right) \\ -\frac{\partial}{\partial x}\left(g u_{1}+\frac{1}{2} u_{2}^{2}\right)\end{array}\right)$,

where $u=\left(u_{1}, u_{2}\right) \in D$. The operator $f$ is $\mathbf{d}$ homogeneous of degree $\nu=1$ on the set

$\Omega=\left\{\left(u_{1}, u_{2}\right) \in D: u_{1}(0) u_{2}(0)=0 ; u_{1}(1) u_{2}(1)=u_{1}^{\frac{3}{2}}(1)\right\}$ with respect to the weighted dilation $\mathbf{d}(s) u=$ $\left(e^{2 s} u_{1}, e^{s} u_{2}\right)$, where $u=\left(u_{1}, u_{2}\right) \in \mathbb{C}([0,1], \mathbb{R}) \times$ $\mathbb{C}([0,1], \mathbb{R})$ and $s \in \mathbb{R}$. Indeed,

$$
\begin{gathered}
f(\mathbf{d}(s) u)=\left(\begin{array}{c}
-\frac{\partial}{\partial x}\left(e^{2 s} u_{1} e^{s} u_{2}\right) \\
-\frac{\partial}{\partial x}\left(g e^{2 s} u_{1}+\frac{1}{2}\left[e^{s} u_{2}\right]^{2}\right)
\end{array}\right)= \\
\left(\begin{array}{c}
-e^{3 s} \frac{\partial}{\partial x}\left(u_{1} u_{2}\right) \\
-e^{2 s} \frac{\partial}{\partial x}\left(g u_{1}+\frac{1}{2} u_{2}^{2}\right)
\end{array}\right)=e^{s} d(s) f(u) .
\end{gathered}
$$

Finally, the equality $(d(s) u)(x)=$ $\left(e^{2 s} u_{1}(x), e^{s} u_{2}(x)\right), x \in \mathbb{R}$ implies that for any $u \in \Omega$ one has $d(s) u \in \Omega$, i.e. the set $\Omega$ is $\mathbf{d}$-homogeneous.

Remark 1: If the operator $g: D \subset \mathbb{B} \rightarrow \mathbb{B}$ is dhomogeneous, then the set

$$
\operatorname{Ker}(g)=\{u \in D: g(u)=0\}
$$

is $\mathbf{d}$-homogeneous. Indeed, if $u \in \operatorname{Ker}(g)$ then $g(\mathbf{d}(s) u)=$ $e^{\nu s} \mathbf{d}(s) g(u)=0$, i.e. $\mathbf{d}(s) u \in \operatorname{Ker}(g)$. So, the kernel of homogeneous operator is $\mathbf{d}$-homogeneous set.

\section{Solutions to Homogeneous Evolution Equations}

This subsection studies some properties of solutions to homogeneous evolution equations. The next theorem provides the most important scalability property of solutions.
Its proof is given for mild solutions of (1), (2), i.e. $u(t, \varphi)=$ $\lim _{\varepsilon \rightarrow 0} u^{\varepsilon}(t, \varphi)$ uniformly on $t$ in a finite interval $[0, T)$, where $u^{\varepsilon}(t, \varphi)$ is a so-called $\varepsilon$-solution:

$$
u^{\varepsilon}(0, \varphi)=u_{0}=\varphi, \quad u^{\varepsilon}(t, \varphi)=u_{i} \in \Omega \text { for } t \in\left[t_{i}, t_{i+1}\right)
$$

and

$$
\frac{u_{i+1}-u_{i}}{t_{i+1}-t_{i}}=f\left(u_{i}\right) \text { for } i=0,1 \ldots, k-1, t_{k}=T,
$$

where $t_{i+1}-t_{i} \leq \varepsilon$ for $i=0, \ldots, k-1$. The proof can be easily repeated for classical solutions, strong or weak solutions.

Theorem 1 (On Homogeneous Dilation of Solutions):

Let an operator $f: \Omega \subset \mathbb{B} \rightarrow \mathbb{B}$ be d-homogeneous of degree $\nu \in \mathbb{R}$ and $u(\cdot, \varphi):[0, T) \rightarrow \Omega$ be a solution of the Cauchy problem (1), (2).

Then for any $s \in \mathbb{R}$ the function $u_{s}:\left[0, \frac{T}{e^{\nu s}}\right) \rightarrow \Omega$ defined by the equality $u_{s}(\tau)=\mathbf{d}(s) u\left(e^{\nu s} \tau, \varphi\right)$ is a solution of the evolution equation (1) with the initial condition $u(0)=$ $\mathbf{d}(s) \varphi$.

Proof. Let $u^{\varepsilon}(\cdot, \varphi)$ be an arbitrary $\varepsilon$-solution of the Cauchy problem (1), (2). For an arbitrary $s \in \mathbb{R}$ let us construct a function $u_{s}^{\varepsilon}:\left[0, \frac{T}{e^{\nu s}}\right) \rightarrow \Omega$ by means of the following relation $u_{s}^{\varepsilon}(\tau)=d(s) u^{\varepsilon}\left(e^{\nu s} \tau, \varphi\right)$, where $\tau \in\left[0, \frac{T}{e^{\nu s}}\right)$.

Let us denote $\tau_{i}=e^{-\nu s} t_{i}$ and $u_{s} i=\mathbf{d}(s) u_{i} \in \Omega$. On the one hand, we have

$$
\frac{u_{s i+1}-u_{s i}}{\tau_{i+1}-\tau_{i}}=e^{\nu s} \mathbf{d}(s) \frac{u_{i+1}-u_{i}}{t_{i+1}-t_{i}} .
$$

On the other hand, the homogeneity of the operator $f$ provides $f\left(\mathbf{d}(s) u_{i}\right)=f\left(u_{s i}\right)=e^{\nu s} \mathbf{d}(s) f\left(u_{i}\right)$. Hence, we derive $\frac{u_{s i+1}-u_{s i}}{\tau_{i+1}-\tau_{i}}=f\left(u_{s i}\right)$, i.e. the function $u_{s}^{\varepsilon}$ is $\varepsilon$-solution of the evolution equation (1) with the initial condition $u(0)=$ $\mathbf{d}(s) \varphi$.

Finally, since $\|\mathbf{d}(s)\|<+\infty$ for any $s \in \mathbb{R}$ independently on $\tau$, then the inequality $\left\|u_{s}^{\varepsilon}(\tau)-u_{s}(\tau)\right\| \leq\|\mathbf{d}(s)\|_{\mathcal{L}}$. $\left\|u^{\varepsilon}(t, \varphi)-u(t, \varphi)\right\|$ implies that $u_{s}(\tau)=\lim _{\varepsilon \rightarrow 0} u_{s}^{\varepsilon}(\tau)$ uniformly on $\tau$ in the interval $\left[0, \frac{T}{e^{\nu s}}\right)$.

Note that under conditions of Theorem 1 the function $u_{s}^{T^{\prime}}$ : $\left[0, \frac{T-T^{\prime}}{e^{\nu s}}\right) \rightarrow \Omega$ defined by $u_{s}^{T^{\prime}}(\tau)=d(s) u\left(T^{\prime}+e^{\nu s} \tau, \varphi\right)$ is the solution of the evolution equation (1) with the initial value $d(s) u\left(T^{\prime}, \varphi\right)$. If the solution $u(\cdot, \varphi)$ is defined on $[0,+\infty)$, then Theorem 1 holds for any finite $T>0$ and $u_{s}^{T^{\prime}}(\cdot)$ is also defined on $[0,+\infty)$.

Theorem 1 yields several corollaries, which expand the local properties of the solutions making them global. For instance, Theorem 1 and Proposition 2 immediately imply the following

Corollary 1 (On Existence and Prolongation of Solutions): Let the operator $f: \Omega \subset \mathbb{B} \rightarrow \mathbb{B}$ be a d-homogeneous operator on the open set $\Omega \subset \mathbb{B}$.

If there exists a set $M \subset \Omega: \bigcup_{s \in \mathbb{R}} d(s) M=\Omega \backslash\{0\}$ and the Cauchy problem (1), (2) has a solution $u(\cdot, \varphi):[0, T) \rightarrow \mathbb{B}$ for any $\varphi \in M$ then it has a solution for any $\varphi \in \Omega$.

Moreover, if $T=+\infty$ for all solutions with $\varphi \in M$ then all solutions of the evolution (1) with $\varphi \in \Omega$ exist on $\mathbb{R}_{+}$. 


\section{Stability Properties of Homogeneous Evolution Equations}

This paper paper deals only with strong stability analysis, so the word "strong" is omitted below for simplicity of presentation. Recall that the solution $u_{0}: \mathbb{R}_{+} \rightarrow \Omega$ of the evolution equation (11) is said to be Lyapunov stable if there exists a continuous monotone increasing function $\sigma:[0,+\infty) \rightarrow[0,+\infty), \sigma(0)=0$ and a number $h \in \mathbb{R}_{+}$ such that $\left\|u(t, \varphi)-u_{0}(t)\right\| \leq \sigma\left(\left\|\varphi-u_{0}(0)\right\|\right)$ for all $t \in \mathbb{R}_{+}$ and for any $\varphi \in \Omega:\|\varphi-u(0)\|<h$.

For asymptotic stability of the solution $u_{0}$ we ask for its Lyapunov stability and the local attractivity of $u_{0}$, i.e. $u(t, \varphi) \rightarrow u_{0}(t)$ as $t \rightarrow+\infty$ if $\varphi \in \Omega:\left\|\varphi-u_{0}(0)\right\|<h$, where the number $h \in \mathbb{R}_{+}$defines the domain of attraction.

The solution $u_{0}: \mathbb{R}_{+} \rightarrow \Omega$ of the evolution equation (1) is said to be uniformly asymptotically stable if it is asymptotically stable and, in addition, for any $r \in(0, h)$ and any $\varepsilon \in(0, r)$ there exists $\tilde{T} \in \mathbb{R}_{+}$such that $\| u(t, \varphi)-$ $u_{0}(t) \|<\varepsilon$ for all $t>\tilde{T}$ and all solutions of the Cauchy problem (1), (2) with $\varphi \in \Omega:\left\|\varphi-u_{0}(0)\right\|<r$.

We refer the reader to [15] for more detailed explanations of different stability properties of evolution equations in Banach spaces.

Below we study the stability property of the zero solution (i.e $u_{0}(\cdot)=0$ ) of the evolution equation (1). Note that the conditions $0 \in \Omega$ and $f(0)=0$ always guarantee existence of the zero solution.

Corollary 2 (On Expansion of Attraction Domain): Let $f: \Omega \subset \mathbb{B} \rightarrow \mathbb{B}$ be $\mathbf{d}$-homogeneous operator and $0 \in \Omega$, $f(0)=0$. If the zero solution of the evolution equation (1) is locally attractive, then it is globally attractive (i.e. $h=+\infty$ ). If, in addition, the zero solution is Lyapunov stable then it is globally asymptotically stable.

The presented corollary can be easily extended to the case of uniform asymptotic stability. Homogeneity also simplifies a finite-time stability [34], [12] analysis of the zero solution of evolution equations. Finite-time stability is the version of the asymptotic stability with finite-time reaching of the stable solution, i.e. for any $\varphi \in \Omega \backslash\{0\}:\left\|\varphi-u_{0}(0)\right\|<h$ there exists $T(\varphi) \in \mathbb{R}_{+}$such $\left\|u(t, \varphi)-u_{0}(t)\right\|=0$ for all $t \geq T(\varphi)$.

To the best of our knowledge, the next property of homogeneous system has been discovered by V.I. Zubov in 1957 and proven for ordinary differential equations using standard (Euler) homogeneity [43].

Theorem 2 (On finite-time stability): Let $f: \Omega \subset \mathbb{B} \rightarrow \mathbb{B}$ be d-homogeneous operator of negative degree $\nu<0$ and $0 \in \Omega, f(0)=0$. If the zero solution of the evolution equation (1) is uniformly asymptotically stable then it is globally finite-time stable.

Proof. I. By Corollary 2 the local uniform asymptotic stability of homogeneous evolution equation implies the global one. So, for each $\varphi \in \Omega$ there exists $\tilde{T} \in \mathbb{R}$ (probably depended on $\varphi$ ) such that $\|u(t, \varphi)\| \leq 1$ for $t \geq \tilde{T}$.

II. Since the zero solution is globally uniformly asymptotically stable then there exists $T^{\prime} \in \mathbb{R}_{+}$such that $\|u(t, \varphi)\| \leq$ $1 / 2$ for all $t \geq T^{\prime}$ and any $\varphi \in \Omega:\|\varphi\| \leq 1$, where $u(t, \varphi)$ is an arbitrary solution of (1), (2). Proposition 1 implies existence of a number $s>0$ such that $\|\mathbf{d}(s)\|_{\mathcal{L}}=2$.
Let us introduce the following notations:

- $\Delta T_{0}=T^{\prime}$ and $\Delta T_{i}=e^{\nu s} \Delta T_{i-1}$ for $i=1,2, \ldots$;

- $T_{0}=0$ and $T_{i}=T_{i-1}+\Delta T_{i-1}$ for $i=1,2, \ldots$;

- $x_{i}=u\left(T_{i}, \varphi\right)$ for $i=1,2, \ldots$.

Obviously, since $u\left(T_{1}, \varphi\right)=x_{1}$ then $\left\|x_{1}\right\| \leq 1 / 2$. By Theorem 11 the function $u_{1}(\cdot)=\mathbf{d}(s) u\left(T_{1}+e^{\nu s} \cdot \varphi\right)$ is also solution of (1) defined on $\mathbb{R}_{+}$. Moreover, $u_{1}(0)=$ $\mathbf{d}(s) u\left(T_{1}, \varphi\right)=\mathbf{d}(s) x_{1}$ and

$$
\left\|u_{1}(0)\right\| \leq\|\mathbf{d}(s)\|_{\mathcal{L}} \cdot\left\|x_{1}\right\| \leq 1 .
$$

In this case, the uniform asymptotic stability implies

$$
\begin{gathered}
\left\|u_{1}\left(T^{\prime}\right)\right\|=\left\|\mathbf{d}(s) u\left(T_{1}+e^{\nu s} T^{\prime}, \varphi\right)\right\|= \\
\left\|\mathbf{d}(s) u\left(T_{1}+\Delta T_{1}, \varphi\right)\right\|=\left\|\mathbf{d}(s) x_{2}\right\| \leq 1 / 2
\end{gathered}
$$

and $\left\|\mathbf{d}(2 s) x_{2}\right\| \leq\|\mathbf{d}(s)\|_{\mathcal{L}}\left\|\mathbf{d}(s) x_{2}\right\| \leq 1$.

Repeating the same consideration by induction we obtain $\left\|\mathbf{d}(i s) x_{i}\right\| \leq 1$ and

$$
\left\|u\left(T_{i}, \varphi\right)\right\|=\left\|\mathbf{d}(-i s) \mathbf{d}(i s) x_{i}\right\| \leq\|\mathbf{d}(-i s)\|_{\mathcal{L}} \rightarrow 0
$$

as $i \rightarrow \infty$ due to the limit property of the dilation.

It is easy to see that $\Delta T_{i}=T^{\prime} e^{i \nu s}$ and for $\nu<0$ we obtain

$$
T_{i}=T^{\prime} \sum_{n=0}^{i-1} e^{n \nu s} \rightarrow \frac{T^{\prime}}{1-e^{\nu s}} \text { as } i \rightarrow \infty .
$$

In other words, $u(t, \varphi) \rightarrow 0$ as $t \rightarrow \frac{T^{\prime}}{1-e^{\nu s}}$, where $s \in \mathbb{R}_{+}$is such that $\|\mathbf{d}(s)\|_{\mathcal{L}}=2$ and $\varphi \in \Omega:\|\varphi\| \leq 1$.

This theorem is very useful for analysis of the convergence rate of homogeneous systems. The related problems appear in control theory (for models represented by ordinary differential equations see, for example, [11], [27], [25], [32]).

Let us present an example of finite-time stable homogeneous evolution equations with negative degree of homogeneity.

- Fast Diffusion Equation. The partial differential equation of the form

$$
\frac{\partial v}{\partial t}+\Delta\left(v^{\alpha}\right)=0, \quad \alpha \in(0,1),
$$

where $\Delta$ is the Laplace operator, $v$ is a scalar nonnegative function of the time $t \in \mathbb{R}_{+}$and the space $x \in \mathbb{R}^{n}$ variables, is known as Fast Diffusion Equation [36], [10], [13], which occurs in modeling of plasmas. The considered equation is usually studied with the homogeneous Dirichlet conditions

$$
v(t, x)=0 \text { for } x \in \partial M,
$$

where $M \subset \mathbb{R}^{n}$ is a bounded connected domain with a smooth boundary. The considered system was studied in [13] under the assumption $\frac{[n-2]_{+}}{n+2}<\alpha<1$, where $[\cdot]_{+}$is the projector to $\mathbb{R}_{+} \cup\{0\}$, which was required for existence of a weak solution for any nonnegative initial condition $v(0, x)=u_{0}(x), x \in M$, where $u_{0} \in$ $\mathbb{L}^{p}(M, \mathbb{R}), p \geq 1$.

It is easy to check that the corresponding evolution equation (1) is homogeneous. Indeed, it has operator $f: D \subset \mathbb{L}^{1}(M, \mathbb{R}) \rightarrow \mathbb{L}^{1}(M, \mathbb{R})$ defined by 
$f(u)=-\Delta\left(u^{\alpha}\right)$ using weak derivatives, where $D=$ $\mathbb{L}^{1}\left(M, \mathbb{R}_{+}\right)$. The operator $f$ is d-homogenenous of negative degree $\alpha-1$ on $\mathbb{L}^{1}(M, \mathbb{R})$ with the uniform dilation $\mathbf{d}(s)=e^{s}$, where $s \in \mathbb{R}$. Indeed,

$f(d(s) u)=-\Delta\left(\left(e^{s} v\right)^{\alpha}\right)=-e^{\alpha s} \Delta\left(v^{s}\right)=e^{(\alpha-1) s} d(s) f(u)$

for any $s \in \mathbb{R}$. Therefore, if the zero solution of fast diffusion equation is asymptotically stable then it is finite-time stable. In [13] finite-time stability has been proven without any homogeneous arguments. This example shows that homogeneity simplifies the qualitative analysis of the stability.

\section{Conclusions}

The notion of $\mathbf{d}$-homogeneous evolution equation introduced in this paper can be considered as a certain analog of geometric homogeneity well-known for finite-dimensional vector fields. We believe that the obtained results about stability and scalability properties of homogeneous evolution equations provide a background required for expansion of homogeneous methods (like Input-to-State Stability or Implicit Lyapunov Function Methods) to evolution equations in a Banach space. The d-homogeneity in this case will play an important role in the analysis and design of fast (finite-time) control and observation algorithms for distributed parameter systems.

\section{REFERENCES}

[1] M. Ablowitz and P. Clarkson. Solutions, Nonlinear Evolution Equations and Inverse Scattering. Cambridge University Press, 1991

[2] F. Alabau-Boussouira, V. Perrollaz, and L. Rosier. Finite-time stabilization of a network of strings.

[3] V. Andrieu, L. Praly, and A. Astolfi. Homogeneous Approximation, Recursive Observer Design, and Output Feedback. SIAM Journal of Control and Optimization, 47(4):1814-1850, 2008.

[4] L. Auslander, L. Green, and F. Hahn. Flows on homogeneous spaces. Princeton University Press, 1963.

[5] A. Bacciotti and L. Rosier. Lyapunov Functions and Stability in Control Theory. Springer, 2nd edition, 2005.

[6] V. Barbu. Nonlinear Differential Equations of Monotone Types in Banach Spaces. Springer, 2010.

[7] P. Benilan and M. Crandall. Regularizing effects of homogeneous evolution equations. In Contributions to Analysis and Geometry, 1981.

[8] E. Bernuau, D. Efimov, W Perruquetti, and A. Polyakov. On homogeneity and its application in sliding mode control. Journal of The Franklin Institute, 351(4):1866-1901, 2014.

[9] E. Bernuau, A. Polyakov, D. Efimov, and W. Perruquetti. Verification of ISS, iISS and IOSS properties applying weighted homogeneity. System \& Control Letters, 62(12):1159-1167, 2013.

[10] J.G. Berryman and C.J. Holland. Stability of the separable solution for fast diffusion. Archive for Rational Mechanics and Analysis, 74(4):379-388, 1980.

[11] S. P. Bhat and D. S. Bernstein. Geometric homogeneity with applications to finite-time stability. Mathematics of Control, Signals and Systems, 17:101-127, 2005.

[12] S.P. Bhat and D.S. Bernstein. Finite time stability of continuous autonomous systems. SIAM J. Control Optim., 38(3):751-766, 2000.

[13] M. Bonforte, G. Grillo, and J. L. Vazquez. Behaviour near extinction for the fast diffusion equation on bounded domains. Journal de Mathématiques Pures et Appliquées, 97:1-38, 2012.

[14] J.-M. Coron, B. Andrea-Novel, and G. Bastin. A strict Lyapunov function for boundary control of hyperbolic systems of conservation laws. IEEE Transactions on Automatic Control, 52(1):2-11, 2007.

[15] L. Dalecky and M. G. Krein. Stability of Solutions of Differential Equations in Banach Spaces. American Mathematical Society, Providence, Rhode Island, 1974.
[16] D. Efimov and W. Perruquetti. Oscillations conditions in homogenous systems. In Proc. IFAC NOLCOS Symp., pages 1379-1384, 2010.

[17] W. Hahn. Stability of Motion. Springer-Verlag Berlin Heidelberg, New York, 1967.

[18] V.T. Haimo. Finite time controllers. SIAM Journal of Control and Optimization, 24(4):760-770, 1986.

[19] H. Hermes. Nilpotent approximations of control systems and distributions. SIAM Journal of Control and Optimization, 24(4):731, 1986.

[20] Y. Hong. $\mathrm{H}_{\infty}$ control, stabilization, and input-output stability of nonlinear systems with homogeneous properties. Automatica, 37(7):819$829,2001$.

[21] L.S. Husch. Topological characterization of the dilation and the translation in frechet spaces. Mathematical Annals, 190:1-5, 1970.

[22] M. Kawski. Geometric homogeneity and stabilization. In Arthur Krener and David Mayne, editors, Proc. IFAC Nonlinear Control Symposium, pages 164-169, Lake Tahoe, CA, 1995.

[23] V. V. Khomenuk. On systems of ordinary differential equations with generalized homogenous right-hand sides. Izvestia vuzov. Mathematica., 3(22):157-164, 1961 (in Russian).

[24] D. J. Korteweg and G. de Vries. On the change of form of long waves advancing in a rectangular canal, and on a new type of long stationary waves. Philosophical Magazine, 39(240):422-443, 1895.

[25] A. Levant. Homogeneity approach to high-order sliding mode design Automatica, 41(5):823-830, 2005.

[26] H. Nakamura. Positive definiteness of generalized homogeneous functions. In 9th Symposium on Nonlinear Control Systems, Toulouse, France, pages 552-557, 4-6 September 2013.

[27] Y. Orlov. Finite time stability and robust control synthesis of uncertain switched systems. SIAM Journal of Control and Optimization, 43(4):1253-1271, 2005.

[28] V. Perrollaz and L. Rosier. Finite-time stabilization of $2 \times 2$ hyperbolic systems on tree-shaped networks. SIAM Journal of Control and Optimization, 52(1):143-163, 2014.

[29] W. Perruquetti, T. Floquet, and E. Moulay. Finite-time observers: application to secure communication. IEEE Transactions on Automatic Control, 53(1):356-360, 2008

[30] A. Polyakov, D. Efimov, and W. Perruquetti. Sliding mode control design for mimo systems: Implicit lyapunov function approach. In European Control Conference (ECC), pages 2612-2617, 2014

[31] A. Polyakov, D. Efimov, and W. Perruquetti. Finite-time and fixedtime stabilization: Implicit lyapunov function approach. Automatica, 51(1):332-340, 2015

[32] A. Polyakov, D. Efimov, and W. Perruquetti. Finite-time stabilization using implicit lyapunov function technique,. In 9th Symposium on Nonlinear Control Systems, Toulouse, France, pages 140-145, 4-6 September 2013.

[33] L. Rosier. Homogeneous Lyapunov function for homogeneous continuous vector field. Systems \& Control Letters, 19:467-473, 1992.

[34] E. Roxin. On finite stability in control systems. Rendiconti del Circolo Matematico di Palermo, 15:273-283, 1966.

[35] E.P. Ryan. Universal stabilization of a class of nonlinear systems with homogeneous vector fields. Systems \& Control Letters, 26:177-184, 1995.

[36] E.S. Sabinina. A class of non-linear degenerating parabolic equations. Soviet Methematics Doklady, 148:495-498, 1962.

[37] J. A. Sanders and J. P. Wang. On the ingerability pf homogeneous scalar evolution equations. Journal of Differential Equations, 147(2):410-434, 1998

[38] R. Sepulchre and D. Aeyels. Homogeneous Lyapunov Functions and Necessary Conditions for Stabilization. Mathematics of Control, Signals and Systems, 9:34-58, 1996.

[39] Y. Shang, D. Liu, and G. Xu. Super-stability and the spectrum of onedimensional wave equations on general feedback controlled networks. IMA Journal of Mathematical Control and Information, 31:73-99, 2014.

[40] V. Sokolov and T. Wolf. Classification of integrable polynomial vector evolution equations. Journal of Physics A, 2001.

[41] Y. Zhang and G. Xu. Exponential and super stability of a wave network. Acta Applicandae Mathematicae, 124(1):19-41, 2012.

[42] S. Zheng. Nonlinear Evolution Equations. CRC Press, 2004

[43] V. I. Zubov. Methods of A.M. Lyapunov and Their Applications. Noordhoff, Leiden, 1964.

[44] V.I. Zubov. On systems of ordinary differential equations with generalized homogenous right-hand sides. Izvestia vuzov. Mathematica., $1: 80-88,1958$ (in Russian). 